\title{
Velocity Transfer Function In The Right Pulmonary Artery And Impaired Cardiopulmonary Reserve In COPD
}

This article was published in the following Dove Press journal: International Journal of Chronic Obstructive Pulmonary Disease

\author{
Oleg F Sharifov' \\ Thomas S Denney Jr2 \\ J Michael Wells $\mathbb{D}^{1,3,4}$ \\ Gregory A Payne (iD) ${ }^{1,4}$ \\ Swati Gulati ${ }^{1,3}$ \\ Himanshu Gupta ${ }^{5}$ \\ Mark T Dransfield 1,3,4 \\ Steven G Lloyd ${ }^{1,4}$ \\ 'Department of Medicine, University of \\ Alabama at Birmingham (UAB), \\ Birmingham, AL, USA; ${ }^{2}$ Department of \\ Electrical and Computer Engineering, \\ Auburn University, Auburn, AL, USA; \\ ${ }^{3} U A B$ Lung Health Center, Birmingham, \\ AL, USA; ${ }^{4}$ Birmingham VA Medical \\ Center, Birmingham, AL, USA; ${ }^{5}$ Advanced \\ Cardiovascular Imaging, Valley Medical \\ Group, Paramus, NJ, USA
}

Correspondence: Steven G Lloyd

Department of Medicine, University of Alabama at Birmingham, 1808 7th Avenue South, BDB 20I, Birmingham, AL 352940012 , USA

$\mathrm{Tel}+$ I 2059349736

$\mathrm{Fax}+$ I 2059349730

Email slloyd@uabmc.edu

\section{Introduction}

Pulmonary hypertension $(\mathrm{PH})$ is an important prognostic indicator in patients with chronic obstructive pulmonary disease (COPD); however, $\mathrm{PH}$ in these patients is typically mild-to-moderate severity and invasive testing is not typically utilized. ${ }^{1}$ Though echocardiography can be used to screen for $\mathrm{PH}$, it has limited accuracy in measuring pulmonary artery (PA) pressure and often hindered by window limitations in COPD patients. ${ }^{2}$ Cardiovascular magnetic resonance (CMR) does not have window limitations and can accurately evaluate the right ventricular (RV) and PA function. Therefore, there is a growing interest in the use of CMR for the diagnosis and management of $\mathrm{PH}$, including patients with lung diseases. Recently, we have validated a novel non-invasive CMR-derived parameter that, similar to impedance, reflects pulsatile and resistive properties of the PA. ${ }^{3}$ This approach relies on the principle that compliant PA vessel walls cause changes in the velocity profile as it travels through the PA over the cardiac cycle. The frequency-dependent relationship between the input and output velocity profiles is described by a velocity transfer function (VTF), which is the relationship between the frequency spectra of input and output velocity. ${ }^{3}$ In suspected PH patients who underwent right heart catheterization, we found that VTF correlates with invasive PA impedance and the mean high-frequency modulus (MHFM) of VTF correlates with the pulmonary vascular resistance (PVR) and RV remodeling. ${ }^{3}$ Moreover, MHFM $>1$ accurately predicted an increased PVR. ${ }^{3}$ We hypothesized that elevated MHFM of VTF, reflecting increased PVR and pulmonary vascular disease, would be associated with reduced exercise capacity measured by 6-mins walk distance (6MWD) in a COPD cohort.

\section{Materials And Methods}

We prospectively recruited participants with COPD and mild-to-moderate airflow obstruction based on Global Initiative for Chronic Obstructive Lung Disease (GOLD) spirometric staging. ${ }^{4}$ Participants were excluded if they had known cardiac or pulmonary vascular disease. This study was approved by the University of Alabama at Birmingham Institutional Review Board and all participants gave written informed consent (IRB\#110809004). Participants completed clinical evaluation, ${ }^{4}$ postbronchodilator spirometry with measurement of percent predicted forced expiratory volume in 1 -second $(\mathrm{FEV} 1 \%){ }^{5}$ quantitative emphysema as measured by chest 
computed tomography (CT), ${ }^{6}$ 6-mins walk test, ${ }^{7}$ and CMR study. The 6-mins walk test was performed as a part of clinical care, prior to the CMR, and was conducted one time according to ATS standards ${ }^{7}$ on room air without stops for a rest. All participants completed the 6-mins walk test. CMR was performed using a 1.5-T magnetic resonance scanner (GE Signa, Milwaukee, Wisconsin) optimized for cardiac applications. Phase-contrast CMR technique (ECG gated breath-hold fast gradient echo (FGRE) sequence) was used for flow measurements in the right PA (RPA) (Figure 1A). Typical parameters were as follows: field of view, $40 \mathrm{~cm}$; scan matrix, 256x128; encoding velocity, $150 \mathrm{~cm} / \mathrm{s}$; number of excitations, 1; flip angle, $15^{\circ}$; repetition/echo times, $7.8 / 3.2 \mathrm{~ms}$; band width, $\pm 31.25 \mathrm{kHz} ; 8$ views per segment. Thirty-two phases were reconstructed. Contours were drawn and mean velocity-time profiles over a cardiac cycle were computed using CAAS MR Flow 1.2 (Pie Medical Imaging, the Netherlands) and exported to MATLAB 2015a for VTF and MHFM of VTF calculation as previously described (Figure $1 \mathrm{~B}$ and $\mathrm{C}$ ). ${ }^{3}$ Cine CMR (ECG gated breath-hold balanced steady-state fast processing (bSSFP) sequence) was used for measurements of RV and left ventricular (LV) structure and function using endocardial and epicardial contours manually traced on short-axis cine images acquired near end-diastole and end-systole as previously described. ${ }^{3}$ CMR measurements were blinded to the 6-mins walk test, clinical evaluation, spirometry, and CT measurements. Participants were categorized into MHFM $>1$ and $\mathrm{MHFM}<1$ groups. Clinical, cardiopulmonary functional indices, LV and RV function was compared between MHFM groups using Mann-Whitney- $U$ test. The strength of association between MHFM of VTF and 6MWD was studied using Spearman's rank correlation coefficient $(\rho)$. Linear regression models adjusted for FEV1\% were used to measure associations between 6MWD and elevated MHFM $(\mathrm{MHFM}>1)$. All analyses were performed in SPSS v.23 and $\mathrm{P}<0.05$ indicated statistical significance.

\section{Results}

The 21 patients recruited were $60 \pm 9$ (mean \pm standard deviation) years old, $62 \%$ male, with an FEV1\% of 61 $\pm 27 \%$ and LV ejection fraction of $62 \pm 8 \%$. Twelve participants had $\mathrm{MHFM}<1$ (median 0.85 [interquartile range (IQR) 0.75-0.90]) and nine participants had $M H F M>1$ (1.23 [IQR 1.10-1.67]). In MHFM $>1$ group, RV ejection fraction (51\% [IQR 47-64\%]), end diastolic volume index (50 [IQR 45-68] $\mathrm{mL} / \mathrm{m}^{2}$ ), and stroke volume (33 [IQR 23-38] $\mathrm{mL} / \mathrm{m}^{2}$ ) were similar to that in $\mathrm{MFHM}<1$ group
(57\% [IQR 54-61\%] $(\mathrm{P}=0.70), 59$ [IQR 56-67] mL/m $\mathrm{m}^{2}$ $(\mathrm{P}=0.25)$, and $34[\mathrm{IQR} 32-36] \mathrm{mL} / \mathrm{m}^{2}(\mathrm{P}=0.76)$, respectively). LV ejection fraction, LV volumes, and cardiac index were also similar between MHFM groups $(\mathrm{P}>0.1)$. The $\mathrm{MHFM}>1$ group had a larger RV mass/volume ratio and $\mathrm{RV} / \mathrm{LV}$ mass ratio (Figure $2 \mathrm{~A}-\mathrm{C}$ ). There were no between-group differences in LV mass index and LV mass to volume ratio $(\mathrm{P}>0.1)$.

MHFM $>1$ group had numerically worse clinical symptoms (though not reaching statistical significance) assessed by modified Medical Research Council (mMRC) Dyspnea Scale (3 [IQR 2-3] vs. 2 [IQR 0-3] in $M H F M<1$ group, $\mathrm{P}=0.25$ ) and severity of airflow limitation by Global Initiative for Chronic Obstructive Lung Disease (GOLD) criteria for GOLD stage (3 [IQR 2-3] vs 1.5 [IQR 0.5-3] in $\mathrm{MHFM}<1$ group, $\mathrm{P}=0.28$ ). FEV1\% was not statistically different in the $M H F M>1$ versus $M H F M<1$ group $(\mathrm{P}=0.26$, Figure $2 \mathrm{D})$. There were no between-group differences in quantitative emphysema (Figure 2E). In the entire cohort, results of the 6-mins walk test were markedly worse than expected for healthy population, ${ }^{7}$ with $6 \mathrm{MWD}$ of 337 [IQR 296-385] meters. There was a significant inverse correlation between 6MWD and MHFM of VTF (Spearman's $\rho=-0.56, \mathrm{P}=0.009$ ). The $\mathrm{MHFM}>1$ group had shorter 6MWD (293 [IQR 268-329] meters vs. 361 [IQR 343-402] meters in $\mathrm{MHFM}<1$ group, $\mathrm{P}=0.0012$ ) (Figure 2F). In a linear regression model adjusting for FEV1\%, MHFM $>1$ accounted for a $-60.7 \pm 16.7$ meter shorter 6MWD $(\mathrm{P}=0.002)$.

\section{Discussion}

Data from our pilot study show that measuring the MHFM of VTF may have utility in assessing pulmonary vascular disease in COPD. Our findings suggest that MHFM of VTF $>1$ is associated with signs of RV remodeling as well as impaired exercise tolerance as measured by 6MWD in COPD. These observed differences occurred in the absence of differences in LV structure or function, percent of emphysema or FEV1\%. Importantly, we found that the association between MHFM $>1$ and reduced 6MWD was independent from FEV1\%, suggesting that it has additional value beyond lung function in identifying impaired exercise function in COPD. Together, MHFM $>1$ and FEV1\% accounted for $53.5 \%$ of the variability in 6MWD. Our study was limited by the small sample size and the lack of invasive hemodynamic assessments, though it mirrored usual clinical care of these patients with COPD, since they usually do not undergo 
A

MAG
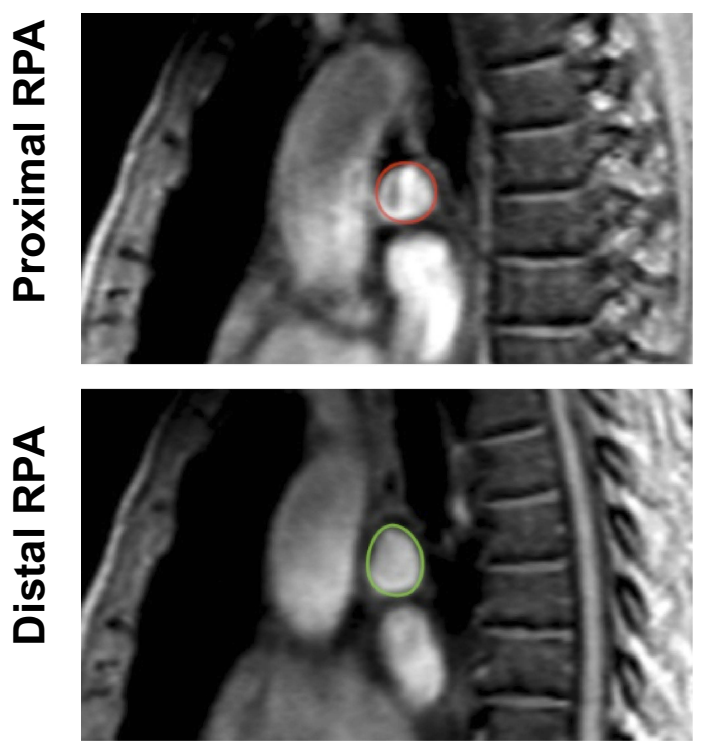

PC
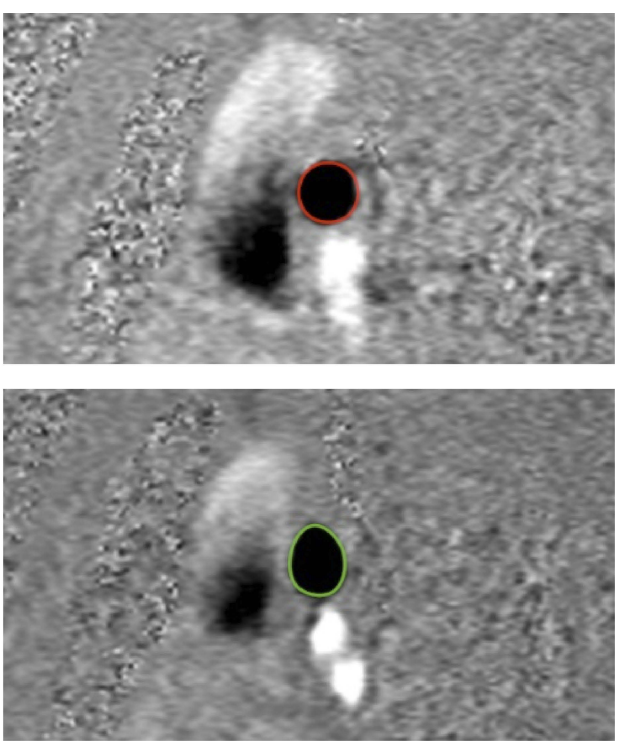

B

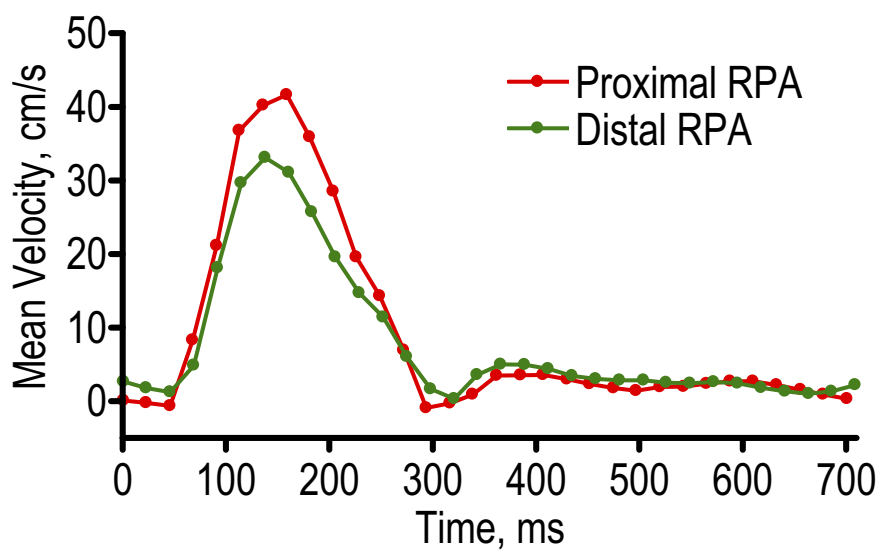

C

$\underline{M H F M}<1$

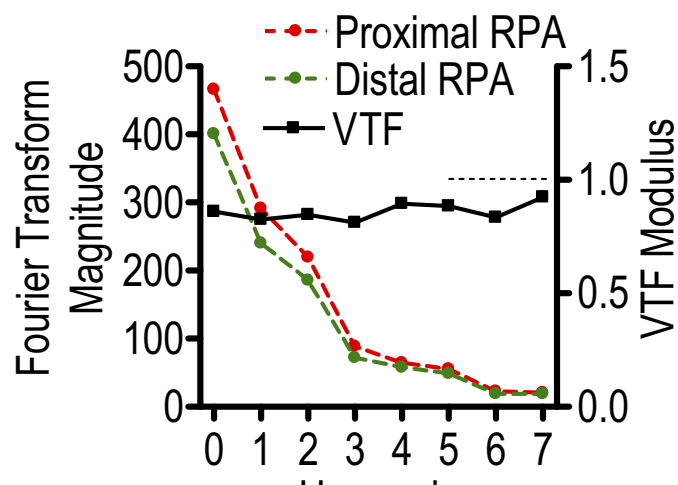

Harmonic
$\underline{M H F M}>1$

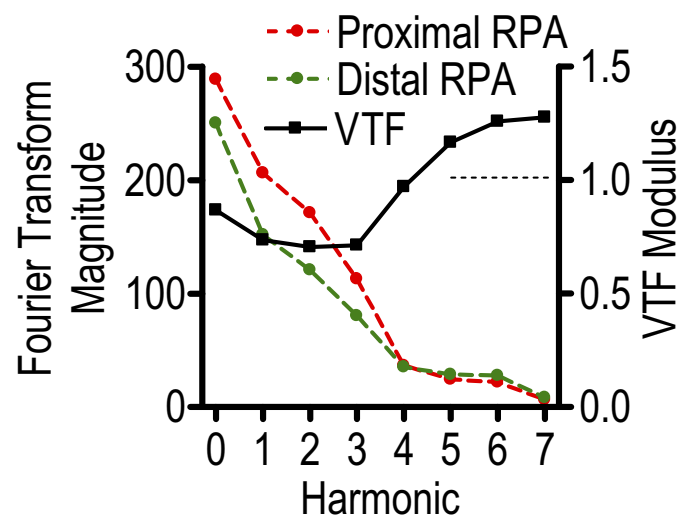

Figure I MHFM of VTF in COPD.

Notes: (A) Representative MAG and PC images from the proximal (red contour) and distal (green contour) portions of the RPA. (B) Representative mean velocity profiles over the cardiac cycle measured in the proximal (red trace) and distal (green trace) portions of the RPA. (C) Representative examples of Fourier transform magnitudes and VTF in subjects with high (>I) and low (<I) MHFM (average modulus for harmonics 5-7).

Abbreviations: MHFM, mean high-frequency modulus; VTF, velocity transfer function; MAG, magnitude; PC, phase-contrast; RPA, right pulmonary artery. 

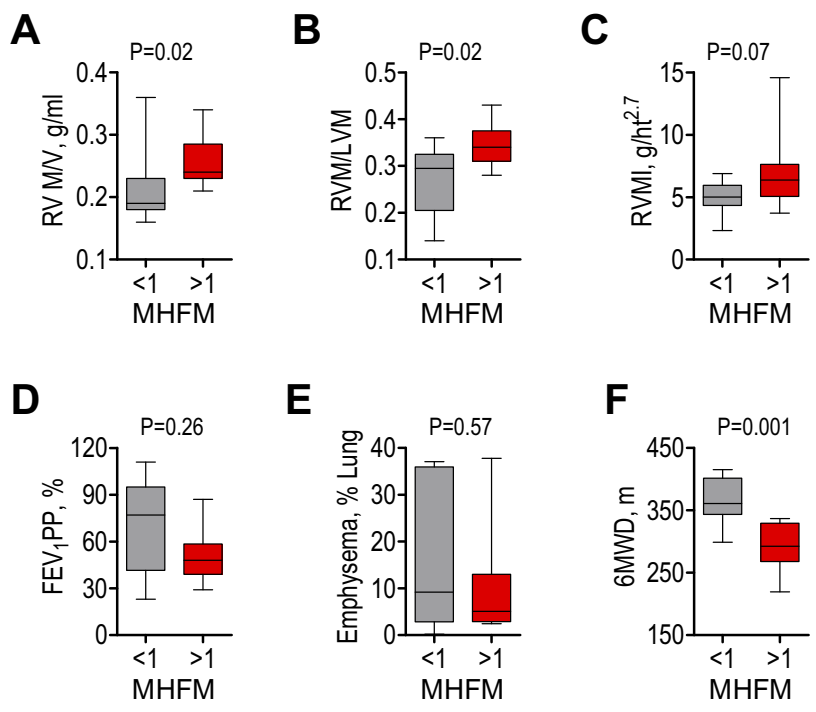

Figure 2 Cardiac and pulmonary measurements in COPD with high $(>I)$ and low (<I) MHFM of VTF.

Notes: High and low MHFM groups had different RVM/V (A), RVM/LVM (B); but did not differ by RVMI (C), FEV , PP (D), or emphysema (E). However, 6MWD was lower in the high MHFM group (F).

Abbreviations: MHFM, mean high-frequency modulus; VTF, velocity transfer function; RVM/V, right ventricular mass to volume ratio; RVM/LVM, ratio of right and left ventricular mass; RVMI, right ventricular mass index; FEV, PP, percent predicted forced expiratory volume in I-second; 6MWD, 6-mins walk distance.

right-sided heart catheterization due to the lack of therapeutic options specific for this population. Our findings should be validated in larger COPD cohorts.

In this proof of concept study, we excluded patients with severe COPD as we were interested in whether VTF could be used as a tool to identify the pulmonary vascular abnormalities in the absence of severe parenchymal destruction and chronic hypoxia. The investigation of VTF utility in more advanced lung disease is therefore needed. In our study, VTF was measured only under resting conditions, yet the measurement of VTF with exercise may provide additional diagnostic power, and thus should be evaluated in future studies. Because we measured VTF in the RPA, any heterogeneity in vessel impedance and stiffness in the pulmonary vasculature could be missed by this technique. Further research is needed to utilize VTF in the main PA and left PA for assessment of heterogeneity in vessel impedance.

\section{Conclusion}

In conclusion, measurement of the mean high-frequency modulus (MHFM) of the VTF, a non-invasive marker of PVR, is associated with impairments in exercise capacity and RV remodeling in a cohort of mild-to-moderate
COPD. Therefore, VTF could be a valuable tool for characterizing pulmonary vascular disease among COPD patients. However, further research is required for accurate evaluation of the VTF application in COPD.

\section{Disclosure}

The University of Alabama at Birmingham (UAB) and Auburn University co-own the patent rights to the VTF method (Gupta and Denney are coinventors). Gupta and Denney are stakeholders in Arcus-Med, LLC, which has licensed exclusive patent rights to the VTF method from $\mathrm{UAB}$ and AU. Gupta and Denney were $10 \%$ owners of Arcus-Med, LCC, during the conduct of the study. In addition, Gupta and Denney have applied for a patent for the VTF method. Gupta is the Chief Medical Officer, and Denney is the Chief Scientific Officer of Arcus-Med, LLC. Wells reports grants from NIH/NHLBI (K08 HL123940) and NIH/NCATS (UL1TR003096), during the conduct of the study; grants, personal fees from GSK, personal fees from AstraZeneca, grants from Bayer, personal fees from BI, grants, personal fees from Mereo BioPharma, outside the submitted work. Dransfield reports grant from NIH/ NHLBI (1K24HL140108), during the conduct of the study; grants from the American Lung Association, Department of Defense, and Department of Veterans Affairs; personal fees from AstraZeneca, GlaxoSmithKline, Mereo, PneumRx/BTG, Pulmonx and Quark; contracted clinical trials from AstraZeneca, Boehringer Ingelheim, Boston Scientific, Gala, GlaxoSmithKline, Nuvaira, PneumRx/BTG, Pulmonx outside the submitted work. The authors report no other conflicts of interest in this work.

\section{References}

1. Minai OA, Chaouat A, Adnot S. Pulmonary hypertension in COPD: epidemiology, significance, and management: pulmonary vascular disease: the global perspective. Chest. 2010;137(6 Suppl):39S-51S. doi:10.1378/chest.10-0087

2. Arcasoy SM, Christie JD, Ferrari VA, et al. Echocardiographic assessment of pulmonary hypertension in patients with advanced lung disease. Am J Respi Crit Care Med. 2003;167(5):735-740. doi:1 0.1164/rccm.200210-1130OC

3. Gupta A, Sharifov OF, Lloyd SG, et al. Novel non-invasive assessment of pulmonary arterial stiffness using velocity transfer function. $J$ Am Heart Assoc. 2018;7:e00959. doi:10.1161/JAHA.118. 009459

4. Vogelmeier CF, Criner GJ, Martinez FJ, et al. Global strategy for the diagnosis, management, and prevention of chronic obstructive lung disease 2017 report. GOLD executive summary. Am J Respir Crit Care Med. 2017;195(5):557-582. doi:10.1164/rccm.2017010218PP 
5. Miller MR, Hankinson J, Brusasco V, et al. Standardisation of spirometry. Eur Respir J. 2005;26(2):319-338. doi:10.1183/090319 36.05.00034805

6. Muller NL, Staples CA, Miller RR, Abboud RT. "Density mask". An objective method to quantitate emphysema using computed tomography. Chest. 1988;94(4):782-787. doi:10.1378/chest.94.4.782
7. ATS statement: guidelines for the six-minute walk test. ATS committee on proficiency standards for clinical pulmonary function laboratories. Am J Respirand Crit Care Med. 2002;166(1):111-117. doi:10.1164/ ajrccm.166.1.at1102

\section{Publish your work in this journal}

The International Journal of COPD is an international, peer-reviewed journal of therapeutics and pharmacology focusing on concise rapid reporting of clinical studies and reviews in COPD. Special focus is given to the pathophysiological processes underlying the disease, intervention programs, patient focused education, and self management protocols. This journal is indexed on PubMed Central, MedLine and CAS. The manuscript management system is completely online and includes a very quick and fair peer-review system, which is all easy to use. Visit http://www.dovepress.com/testimonials.php to read real quotes from published authors.

Submit your manuscript here: https://www.dovepress.com/international-journal-of-chronic-obstructive-pulmonary-disease-journal 